\title{
Local Lyapunov Functions for Consensus in Switching Nonlinear Systems
}

\author{
Johan Thunberg, Member, IEEE, Xiaoming Hu, Member, IEEE, Jorge Gonçalves, Member, IEEE
}

\begin{abstract}
This note presents two theorems on asymptotic state consensus of continuous time nonlinear multi-agent systems. The agents reside in $\mathbb{R}^{m}$ and have switching interconnection topologies. Both the first theorem, formulated in terms of the states of individual agents, and the second theorem, formulated in terms of the pairwise states for pairs of agents, can be interpreted as variants of Lyapunov's second method. The two theorems complement each other; the second provides stronger convergence results under weaker graph topology assumptions, whereas the first often can be applied in a wider context in terms of the structure of the right-hand sides of the systems. The second theorem also sheds some new light on well-known results for consensus of nonlinear systems where the right-hand sides of the agents' dynamics are convex combinations of directions to neighboring agents. For such systems, instead of proving consensus by using the theory of contracting convex sets, a local quadratic Lyapunov function can be used.
\end{abstract}

Index Terms-Consensus, multi-agent systems, nonlinear systems, switched systems.

\section{INTRODUCTION}

In dynamical systems, state convergence to a desired set, e.g., a set of equilibrium points, appears in many important applications. Applications range from the platooning problem [1], where adjacent cars in the formation should keep a constant distance between each other, to wireless cellular networks, where the power usage in the cell phones should converge to a constant low level - power rushes shall be avoided [2]. In biology, concentrations of species governed by mass-action kinetics, should converge to the set of equilibria [3].

Complex nonlinear multi-agent systems have a large number of states and conditions for convergence is often non-trivial. One of the most studied problems, both in terms of the number of applications and the number of theoretical results, is the consensus set, i.e., the set where all the states are equal [4] [11]. In this case, consensus (rendezvous or state agreement) refers to state convergence to the consensus set in which all the states are equal. During the last decade a vast amount of publications addressed consensus. A few examples are the following books [5], [12] and surveys [13]-[15], addressing the subject from different perspectives. The interest in the consensus problem stems on the one hand from the properties of the set - among other things, it is a linear subspace -

Johan Thunberg and Jorge Gonçalves are with the Luxembourg Centre for Systems Biomedicine, Luxembourg, \{johan.thunberg, Jorge.goncalves\}@uni.lu. Xiaoming Hu is with KTH Royal Institute of Technology, Sweden, hu@math.kth.

The authors gratefully acknowledge the financial support form the Fonds National de la Recherche, Luxembourg (FNR8864515). and on the other hand from the applicability of the results. For example, it can be shown that the problem of reaching a formation in many cases can equivalently be posed as a consensus problem [16].

Depending on the particular application, nonlinear multiagent systems can be described in discrete time [17], [18] or continuous time [19]. For linear dynamics, many results have centered around graph theoretic concepts such as the graph Laplacian matrix and its importance for the convergence [4], [5], [20]. Moreover, necessary conditions for consensus have been formulated for homogeneous linear systems [21]. In [22] an algorithm is provided for minimum time consensus.

This work addresses consensus for continuous time multiagent systems with switching interconnection topologies. In many applications, such as networks of cameras [23] or satellites in space [24] equipped with cameras, the topologies change over time. In these scenarios cameras (or agents) that are visible might, as time progresses, fall outside the field of view. For the systems we study, the right-hand side of the system dynamics switches between a finite set of functions that are piece-wise continuous in time and uniformly Lipschitz in state on some compact region containing the origin. Similar to other papers on switching systems, we assume there is a positive lower bound on the dwell-time between consecutive switches [25], [26]. We also require, in order to consider piecewise continuously time-varying right-hand sides, an upper bound on the dwell-time.

In the spirit of Lyapunov's second method, this paper presents two theorems for consensus. The two theorems differ in the following sense: the first is formulated for the individual states of the agents, whereas the second is formulated for pairs of states. The theorems can be combined to show consensus under the assumptions in [9], [25], [26]. However, there are examples where those assumptions do not hold, but the proposed theorems still can be applied.

Both theorems introduce local Lyapunov-like functions, guaranteeing consensus in multi-agent systems. The benefits of using these functions - compared to traditional approaches of using common Lyapunov functions - is that the convergence analysis can be conducted on a local level for individual agents or pairs of agents. Hence, the convergence analysis can be seen as distributed. If the conditions in the first/second theorem are satisfied, then the consensus set is attractive/uniformly asymptotically stable, respectively. While the second theorem provides stronger guarantees for the convergence, the first theorem can often be applied in a wider context. The type of convergence considered in this work is defined relative to compact invariant sets. 
The paper proceeds as follows. Section II introduces the system dynamics and the definitions. Section III provides the main results, i.e., the two main theorems. Section IV provides two examples that illustrate how both theorems can be used. Section V provides outlines of the proofs of the two main theorems. The important steps of the proofs are described. Also, the complete proofs of two support lemmas are contained in this section.

\section{PRELIMINARIES}

\section{A. Notation}

A vector $x$ that belongs to $\mathbb{R}^{m}$ is a column vector. If nothing else is mentioned, $\|\cdot\|$ is the Euclidean norm. $B_{r, m}\left(x_{0}\right)$ denotes the open ball in $\mathbb{R}^{m}$ centered around $x_{0} \in \mathbb{R}^{m}$ with radius $r>0$ and $\bar{B}_{r, m}\left(x_{0}\right)$ is the closure of said open ball. When $x_{0}$ is omitted and the notations $B_{r, m}$ respective $\bar{B}_{r, m}$ are used, it holds that $x_{0}=0$.

\section{B. System dynamics}

Consider a multi-agent system with $n$ agents. The state of agent $i$ at time $t$ is $x_{i}(t) \in \mathbb{R}^{m}$, and the state of the entire system is $x(t)=\left[x_{1}^{T}(t), x_{2}^{T}(t), \ldots, x_{n}^{T}(t)\right]^{T}$. The dynamics is

$$
\left\{\begin{array}{c}
\dot{x}_{1}=f_{1}(t, x), \\
\dot{x}_{2}=f_{2}(t, x), \\
\vdots \\
\dot{x}_{n}=f_{n}(t, x) .
\end{array}\right.
$$

The right-hand side of each agent $i$ has the following structure:

$$
f_{i}(t, x)=\tilde{f}_{\sigma_{i}(t), i}\left(t-\gamma_{\sigma_{i}}(t), x\right)
$$

where

- the functions $\tilde{f}_{l, i} \in \mathcal{F}_{i}$, for all $l \in\left\{1,2, \ldots,\left|\mathcal{F}_{i}\right|\right\}$. The set $\mathcal{F}_{i}$ is a finite set of functions, where each function is continuous in $t$ and Lipschitz in $x$, uniformly with respect to $t$, on some open connected set containing the compact region $\mathcal{D} \subset \mathbb{R}^{m n}$, which contains the origin as an interior point;

- the function $\sigma_{i}(t)$ is piecewise right-continuous and attains values in $\left\{1,2, \ldots,\left|\mathcal{F}_{i}\right|\right\}$;

- the sequence $\left\{\tau_{k}^{i}\right\}$ is a monotonically increasing sequence, such that $\tau_{k}^{i} \rightarrow \infty$ as $k \rightarrow \infty$ and $\tau_{k}^{i} \rightarrow-\infty$ as $k \rightarrow-\infty$, where each $\tau_{k}^{i} \in \mathbb{R}$ is such that for any $k \in \mathbb{Z}$ the function $\sigma_{i}$ is constant on $\left[\tau_{k}^{i}, \tau_{k+1}^{i}\right)$ for all $k$, and

$$
\inf _{k}\left(\tau_{k+1}^{i}-\tau_{k}^{i}\right)>0
$$

- it holds that

$$
\sup _{k}\left(\tau_{k+1}^{i}-\tau_{k}^{i}\right)<\infty
$$

- the function $\gamma_{\sigma_{i}}$ is defined (for finite times $t$ ) by

$$
\gamma_{\sigma_{i}}(t)=\max \left\{\tau_{k}^{i}: \tau_{k}^{i} \leq t, k \in \mathbb{Z}\right\} .
$$

Let $\sigma=\left[\sigma_{1}, \sigma_{2}, \ldots, \sigma_{n}\right]^{T}$ and $\mathcal{F}=\mathcal{F}_{1} \times \mathcal{F}_{2} \times \cdots \mathcal{F}_{n}$. Let $\mathcal{S}_{\mathcal{F}}$ be the set of all possible $\sigma$-functions that can be constructed by $\sigma_{i}$-functions satisfying the conditions above. For agent $i,\left\{\tau_{k}^{i}\right\}$ are the switching times for $\sigma_{i}$, i.e., the points of discontinuity. The $f_{i}$-functions are more general than timeinvariant functions, but the time dependence has a restricted form. At the time $t, f_{i}$ is, besides a function of the state, a function of the time passed since the preceding switching time, see the definition of $\gamma_{\sigma_{i}}(t)$ above.

Each agent $i$ has its own switching signal function $\sigma_{i}$ with a corresponding switching sequence $\left\{\tau_{k}^{i}\right\}$ and there is no dependence between the different $\sigma_{i}$-functions of the agents. Let

$$
\begin{aligned}
& x^{\sigma}\left(t, t_{0}, x_{0}\right) \\
= & {\left[x_{1}^{\sigma_{1}}\left(t, t_{0}, x_{0}\right)^{T}, x_{2}^{\sigma_{2}}\left(t, t_{0}, x_{0}\right)^{T}, \ldots, x_{n}^{\sigma_{n}}\left(t, t_{0}, x_{0}\right)^{T}\right]^{T} }
\end{aligned}
$$

be the solution to (1) with initial state $x_{0}$, initial time $t_{0}$, and switching signal functions given by $\left[\sigma_{1}, \sigma_{2}, \ldots, \sigma_{n}\right]^{T}=\sigma$.

\section{Connectivity}

The dynamical behavior depends on the connectivity between the agents. The connectivity is described by a graph.

Definition 1. A directed graph (or digraph) $\mathcal{G}=(\mathcal{V}, \mathcal{E})$ consists of a set of nodes, $\mathcal{V}=\{1, \ldots, n\}$ and a set of edges $\mathcal{E} \subset \mathcal{V} \times \mathcal{V}$.

Each node in the graph corresponds to a unique agent. Let $\mathcal{N}_{i} \in \mathcal{V}$ comprise the neighbor set (sometimes referred to simply as neighbors or neighborhood) of agent $i$, where $j \in \mathcal{N}_{i}$ if and only if $(i, j) \in \mathcal{E}$. It is assumed that $i \in \mathcal{N}_{i}$ i.e., the collection of graphs is restricted to those for which $(i, i) \in \mathcal{E}$ for all $i \in \mathcal{V}$.

Definition 2. A directed path of $\mathcal{G}$ is an ordered sequence of distinct nodes in $\mathcal{V}$ such that any consecutive pair of nodes in the sequence corresponds to an edge in the graph.

Definition 3. An agent $i$ is connected to an agent $j$ if there is a directed path starting in $i$ and ending in $j$.

Definition 4. A digraph is strongly connected if each node is connected to all other nodes.

Definition 5. A digraph is quasi-strongly connected if there exists a rooted spanning tree or a center, i.e., at least one node such that all the other nodes are connected to it.

Definition 6. The time-varying graph corresponding to $\sigma$, denoted by, $\mathcal{G}_{\sigma(t)}$ is defined by using time-varying neighborhoods $\mathcal{N}_{i}^{\sigma_{i}(t)}$ (also written as $\mathcal{N}_{i}(t)$ or $\mathcal{N}_{i}^{\sigma_{i}}(t)$ ), where $i \in \mathcal{N}_{i}^{k} \subset$ $\{1,2, \ldots, n\}$ for all $i \in\{1,2, \ldots, n\}, k \in\left\{1,2, \ldots,\left|\mathcal{F}_{i}\right|\right\}$. The time-varying graph $\mathcal{G}_{\sigma(t)}=\left(\mathcal{V}, \mathcal{E}_{\sigma(t)}\right)$ is defined by

$$
\mathcal{G}_{\sigma(t)}=\left(\mathcal{V}, \mathcal{E}_{\sigma(t)}\right)=\left(\mathcal{V}, \bigcup_{i} \bigcup_{j \in \mathcal{N}_{i}^{\sigma_{i}(t)}}\{(i, j)\}\right)
$$

Definition 7. The union graph of $\mathcal{G}_{\sigma(t)}$ during the time interval $\left[t_{1}, t_{2}\right)$ is defined by

$$
\mathcal{G}\left(\left[t_{1}, t_{2}\right)\right)=\bigcup_{t \in\left[t_{1}, t_{2}\right)} \mathcal{G}_{\sigma(t)}=\left(\mathcal{V}, \bigcup_{t \in\left[t_{1}, t_{2}\right)} \mathcal{E}_{\sigma(t)}\right),
$$

where $t_{1}<t_{2} \leq+\infty$. 
Definition 8. The graph $\mathcal{G}_{\sigma(t)}$ is uniformly (quasi-) strongly connected if there exists a constant $T^{\sigma}>0$ such that the union graph $\mathcal{G}\left(\left[t, t+T^{\sigma}\right)\right)$ is (quasi-) strongly connected for all $t$.

\section{Stability}

The first definition regards stability of the origin. The stability holds for all choices of the $\sigma$ in $\mathcal{S}_{\mathcal{F}}$. We assume that all the balls or regions in the following definition are contained in $\mathcal{D}$. The existence of such regions is assured by the assumption that the point 0 is in the interior of $\mathcal{D}$.

Definition 9. The point $0 \in \mathbb{R}^{m n}$ is uniformly stable for (1) if for $\varepsilon>0$, there is $\delta(\epsilon)>0$ such that

$$
\begin{aligned}
& x^{\sigma}\left(t_{0}, t_{0}, x_{0}\right) \in \bar{B}_{\delta, m n} \Longrightarrow x^{\sigma}\left(t, t_{0}, x_{0}\right) \in \bar{B}_{\epsilon, m n}, \\
& \text { for all } t \geq t_{0}, \sigma \in \mathcal{S}_{\mathcal{F}} .
\end{aligned}
$$

Definition 10. For a set $\mathcal{A} \subset \mathbb{R}^{m n}$, let

$$
\operatorname{dist}(x, \mathcal{A})=\inf _{y \in \mathcal{A}}\|x-y\| .
$$

Definition 11. The state $x(t)$ approaches $\mathcal{A}$ or $x(t) \rightarrow \mathcal{A}$ as $t \rightarrow \infty$, on a subset of $\mathcal{D}$ if for all $\epsilon>0, t_{0}$, and $x_{0}$ in the subset, there exists $T\left(\epsilon, x_{0}, t_{0}\right)$ such that dist $(x(t), \mathcal{A})<\epsilon$ for all $t \geq T$.

Let us proceed with the definition of invariance of a set for the system (1).

Definition 12. A set $\mathcal{A} \subset \mathcal{D}$ is (positively) invariant for the system (1) if for all $t_{0}$, it holds that

$$
x_{0} \in \mathcal{A} \Longrightarrow x^{\sigma}\left(t, t_{0}, x_{0}\right) \in \mathcal{A}
$$

for all $t>t_{0}$ and $\sigma \in \mathcal{S}_{\mathcal{F}}$.

Let

$$
\begin{aligned}
\mathcal{D}^{*}(\tilde{t})= & \left\{x_{0} \in \mathbb{R}^{m n}: x^{\sigma}\left(t, t_{0}, x_{0}\right) \in \mathcal{D}\right. \\
& \text { for all } \left.t_{0}, t \in\left[t_{0}, t_{0}+\tilde{t}\right), \sigma \in \mathcal{S}_{\mathcal{F}}\right\} .
\end{aligned}
$$

Lemma 1. For any $\tilde{t} \in[0, \infty]$, the set $\mathcal{D}^{*}(\tilde{t})$ is compact and the set $\mathcal{D}^{*}(\infty)$ is also positively invariant.

In the definitions of stability of the origin and the definitions of invariance, it was assumed that $\sigma \in \mathcal{S}_{\mathcal{F}}$ is arbitrary, i.e., the statements must hold for any $\sigma \in \mathcal{S}_{\mathcal{F}}$. However, in the definitions of stability of a set, which are to be formulated now, only the case when $\sigma$ is fixed is considered. Thus, in the following definitions $x$ is written instead of $x^{\sigma}$. The state is restricted to be contained in the invariant compact set $\mathcal{D}^{*}(\infty)$. Hence, the stability of a set is only defined in the relative sense, relative to $\mathcal{D}^{*}(\infty)$. In these definitions it is assumed $\mathcal{D}^{*}(\infty)$ is nonempty; later on conditions are provided in order to assure this.

Definition 13. For (1) where $\sigma \in \mathcal{S}_{\mathcal{F}}$, the set $\mathcal{A}$ is

1) stable relative to $\mathcal{D}^{*}(\infty)$ if for all $t_{0}$ and for all $\epsilon>0$, there is $\delta\left(t_{0}, \epsilon\right)>0$ such that for $x_{0} \in \mathcal{D}^{*}(\infty)$ it holds that

$$
\begin{aligned}
& \operatorname{dist}\left(x_{0}, \mathcal{A}\right) \leq \delta \Longrightarrow \\
& \operatorname{dist}\left(x\left(t, t_{0}, x_{0}\right), \mathcal{A}\right) \leq \epsilon \text { for all } t \geq t_{0} .
\end{aligned}
$$

2) uniformly stable relative to $\mathcal{D}^{*}(\infty)$ if it fulfills 1) and $\delta$ as a function of $t_{0}$ is constant;

3) attractive relative to $\mathcal{D}^{*}(\infty)$ if there is $c\left(t_{0}\right)$ such that $x\left(t, t_{0}, x_{0}\right) \rightarrow \mathcal{A}$ as $t \rightarrow \infty$ for all $x_{0} \in \mathcal{D}^{*}(\infty)$ where $\operatorname{dist}\left(x_{0}, \mathcal{A}\right) \leq c$;

4) uniformly attractive relative to $\mathcal{D}^{*}(\infty)$ if it fulfills 3$)$ and $c$ as a function of $t_{0}$ is constant. Furthermore, it must also hold that if $\operatorname{dist}\left(x_{0}, \mathcal{A}\right) \leq c$, then for any $\eta>0$ there is $T(\eta)$ such that

$$
t \geq t_{0}+T(\eta) \Longrightarrow \operatorname{dist}\left(x\left(t, t_{0}, x_{0}\right), \mathcal{A}\right)<\eta \text { for all } t_{0} ;
$$

5) asymptotically stable relative to $\mathcal{D}^{*}(\infty)$ if it fulfills 1) and 3);

6) uniformly asymptotically stable relative to $\mathcal{D}^{*}(\infty)$ if it fulfills 2) and 4);

7) globally uniformly asymptotically stable relative to $\mathcal{D}^{*}(\infty)$, if it fulfills 6 ) and

$$
c=\sup _{y \in \mathcal{D}^{*}(\infty)} \operatorname{dist}(y, \mathcal{A})
$$

8) globally quasi-uniformly attractive relative to $\mathcal{D}^{*}(\infty)$ if $x\left(t, t_{0}, x_{0}\right) \rightarrow \mathcal{A}$ as $t \rightarrow \infty$ for all $x_{0} \in \mathcal{D}^{*}(\infty)$ and all $t_{0}$. Furthermore, for all $\eta>0$ there is $T(\eta)$ such that

$$
\min _{t \in\left[t_{0}, t_{0}+T(\eta)\right]} \operatorname{dist}\left(x\left(t, t_{0}, x_{0}\right), \mathcal{A}\right)<\eta
$$

for all $x_{0} \in \mathcal{D}^{*}(\infty)$ and $t_{0}$.

\section{MAIN RESULTS}

In the following, the set $\mathcal{A}$ as is chosen as the consensus set, i.e.,

$$
\mathcal{A}=\left\{x=\left[x_{1}^{T}, \ldots, x_{n}^{T}\right]^{T} \in \mathbb{R}^{m n}: x_{i}=x_{j} \text { for all } i, j\right\} .
$$

The first assumption below is creating a relationship between the functions in $\mathcal{F}$ and the neighborhoods of the agents. Together with the two assumptions that follow, the assumption is used in the two main theorems.

Assumption 1. For all $i \in\{1,2, \ldots, \mathcal{V}\}$ and $l \in$ $\left\{1,2, \ldots,|\mathcal{F}|_{i}\right\}$ it holds that $\tilde{f}_{l, i}(t, z) \in \mathcal{F}_{i}$ is, besides a function of $t$, only a function of $\left\{z_{j}\right\}_{j \in \mathcal{N}^{l}}$ on $\mathbb{R} \times \mathcal{D}$, where $z=\left[z_{1}^{T}, z_{2}^{T}, \ldots, z_{n}^{T}\right]^{T} \in \mathcal{D}$ and $z_{i} \in \mathbb{R}^{m}$ for all $i$.

Assumption 2. Let $V: \mathbb{R}^{m} \rightarrow \mathbb{R}$ be a continuously differentiable function. The function $V$ fulfills the following:

1) $V$ is positive definite.

2) Suppose $i \in \mathcal{V}, l \in\left\{1,2, \ldots,|\mathcal{F}|_{i}\right\}, \tilde{f}_{l, i} \in \mathcal{F}_{i}$, and $z=\left[z_{1}^{T}, z_{2}^{T}, \ldots, z_{n}^{T}\right]^{T} \in \mathcal{D}$. If $V\left(z_{i}\right) \geq V\left(z_{j}\right)$ for all $j \in \mathcal{N}_{i}^{l}$, it holds that

$$
\nabla V\left(z_{i}\right) \tilde{f}_{l, i}(t, z) \leq 0
$$

for all $t$. 
3) If 2) holds and there is $j \in \mathcal{N}_{i}^{l}$ such that $z_{i} \neq z_{j}$, the inequality in (2) is strict. Furthermore, if the inequality in (2) is not strict for $z$ and some $\bar{t}$, then it holds that $\tilde{f}_{l, i}(t, z)=0$ for all $t$.

Assumption 3. Let $W: \mathbb{R}^{m} \times \mathbb{R}^{m} \rightarrow \mathbb{R}^{+}$be a continuously differentiable function. The function $W$ fulfills the following.

1) $W(x, y)=0$ if and only if $x=y$.

2) Suppose $(i, j) \in \mathcal{V} \times \mathcal{V}, l_{1} \in\left\{1,2, \ldots,|\mathcal{F}|_{i}\right\}, l_{2} \in$ $\left\{1,2, \ldots,|\mathcal{F}|_{j}\right\}, \quad \tilde{f}_{l_{1}, i} \in \mathcal{F}_{i}, \quad \tilde{f}_{l_{2}, j} \in \mathcal{F}_{j}$, and $z=$ $\left[z_{1}^{T}, z_{2}^{T}, \ldots, z_{n}^{T}\right]^{T} \in \mathcal{D}$. If $W\left(z_{i}, z_{j}\right) \geq W\left(z_{k_{1}}, z_{k_{2}}\right)$ for all $k_{1} \in \mathcal{N}_{i}^{l_{1}}$ and all $k_{2} \in \mathcal{N}_{j}^{l_{2}}$, it holds that

$$
\nabla W\left(z_{i}, z_{j}\right)\left[\tilde{f}_{l_{1}, i}^{T}(t, z), \tilde{f}_{l_{2}, j}^{T}(t, z)\right]^{T} \leq 0
$$

for all $t$.

3) Furthermore, if 2) holds, the inequality in (3) is strict if there is $k_{1} \in \mathcal{N}_{i}^{l_{1}}$ such that $z_{i} \neq z_{k_{1}}$, or if there is $k_{2} \in \mathcal{N}_{j}^{l_{2}}$ such that $z_{j} \neq z_{k_{2}}$, and if the inequality in (2) is not strict for $z$ and some $\bar{t}$, then it holds that $\tilde{f}_{l_{1}, i}(t, z)=0$ and $\tilde{f}_{l_{2}, j}(t, z)=0$ for all $t$.

4) If 2) holds, the inequality in (3) is strict only if there is $k_{1} \in \mathcal{N}_{i}^{l_{1}}$ such that $z_{i} \neq z_{k_{1}}$, or if there is $k_{2} \in \mathcal{N}_{j}^{l_{2}}$ such that $z_{j} \neq z_{k_{2}}$.

Lemma 2. Suppose Assumption 2 1) and 2) hold for the function $V$, then 0 is uniformly stable for (1). Furthermore, suppose $\widehat{\beta}_{1}$ and $\widehat{\beta}_{2}$ are class $\mathcal{K}$ functions such that

$$
\widehat{\beta}_{1}(\|y\|) \leq V(y) \leq \widehat{\beta}_{2}(\|y\|),
$$

then for $\epsilon$ such that $\left(\bar{B}_{\epsilon, m}\right)^{n} \subset \mathcal{D}$, it holds that for all $t_{0}$ and $\sigma \in \mathcal{S}_{\mathcal{F}}$, if $\delta(\epsilon)=\widehat{\beta}_{2}^{-1}\left(\widehat{\beta}_{1}(\epsilon)\right)$,

$$
\begin{aligned}
& x_{i}^{\sigma_{i}}\left(t_{0}, t, x_{0}\right) \in \bar{B}_{\delta, m} \text { for all } i \Longrightarrow \\
& x_{i}^{\sigma_{i}}\left(t, t_{0}, x_{0}\right) \in \bar{B}_{\epsilon, m}, \text { for all } i, t \geq t_{0},
\end{aligned}
$$

and $\bar{B}_{\delta, m} \subset \mathcal{D}^{*}(\infty)$.

Remark 1. What we mean when we say that Assumption 2 1) and 2) hold, is that everything in Assumption 2 hold except possibly 3).

Now the two main theorems are presented, after which three remarks explain some of the properties of the theorems. The first theorem is formulated for $V$-functions in Assumption 2, whereas the second theorem is formulated for $W$-functions in Assumption 3. What essentially differ between the two theorems are the assumptions on the convergence rate and the graph topologies - Theorem 2 provides more in both regards. But, there are systems for which it is unclear how Theorem 2 can be used, whereas application of Theorem 1 is straight forward, See Section IV-B. A nice geometric understanding of the differences of the two theorems can be obtained in the classical example where the right-hand sides of the agents' dynamics are convex combinations of the directions to neighboring agents, see Section IV-A and figures 1 and 2. In this case the $V$ and the $W$ functions are chosen as the
Euclidean norms squared of the individual states respective the difference between pairs of states.

Theorem 1. Suppose Assumption 1 and Assumption 2 2), 3) hold and $\sigma \in \mathcal{S}_{\mathcal{F}}$ is such that $\mathcal{G}_{\sigma(t)}$ is uniformly strongly connected. The consensus set $\mathcal{A}$ is globally quasi-uniformly attractive relative to $\mathcal{D}^{*}(\infty)$.

Theorem 2. Suppose Assumption 1 and Assumption 3 hold, and $\sigma \in \mathcal{S}_{\mathcal{F}}$. The consensus set $\mathcal{A}$ is globally uniformly asymptotically stable relative to $\mathcal{D}^{*}(\infty)$ if and only if $\mathcal{G}_{\sigma(t)}$ is uniformly quasi-strongly connected.

Remark 2. If Assumption 1 holds and Assumption 3 1), 2) and 3) hold, Theorem 2 holds provided the phrase "if and only if" is replaced with "if".

Remark 3. Provided Assumption 2 1), 2) hold, we can show that $\mathcal{D}^{*}(\infty)$ is nonempty, and an easy way of guaranteeing that $x_{0} \in \mathcal{D}^{*}(\infty)$ is to use Lemma 2 and let $x_{0} \in\left(\bar{B}_{\delta, m}\right)^{n} \subset \mathcal{D}^{*}(\infty)$. When we know that $\mathcal{D}^{*}(\infty)$ is nonempty and $x_{0} \in \mathcal{D}^{*}(\infty)$, we do not require $V$ to be positive definite in Theorem 1, i.e., it is sufficient that only conditions 2) and 3) hold for $V$ in Assumption 2. This means that we can use one positive definite function $V_{1}$ in Lemma 2 in order to construct a set that is contained in $\mathcal{D}^{*}(\infty)$, and another - not necessarily positive definite function $-V_{2}$ in order to show that $\mathcal{A}$ is attractive in Theorem 1.

\section{EXAMPLES AND INTERPRETATIONS}

In this section two examples of systems on the form (1) are provided for which the theorems are applicable.

\section{A. A convexity assumption}

In this subsection $V$ and $W$ are chosen as the Euclidean norm squared of the states and the relative states, respectively. Under certain conditions, these choices of functions can be used to show a well-known result that, provided the righthand side of each agent's dynamics as an element of the tangent space $T_{x_{i}} \mathbb{R}^{m}$ is inward-pointing [27] relative to the convex hull of its neighbors, the system reaches consensus asymptotically [25], [26]. We define the tangent cone to a convex set $S \in \mathbb{R}^{m}$ at the point $y$ as

$$
\mathcal{T}(y, S)=\left\{z \in \mathbb{R}^{m}: \liminf _{\lambda \rightarrow 0} \frac{\operatorname{dist}(y+\lambda z, S)}{\lambda}=0\right\} .
$$

This definition is provided in [25], and $\xi$ is inward-pointing relative to $S$, where $0 \neq \xi \in T_{y} \mathbb{R}^{m}\left(T_{y} \mathbb{R}^{m}\right.$ is the tangent space of $\mathbb{R}^{m}$ at the point $y$ ), if $\xi$ belongs to the relative interior of $\mathcal{T}(y, S)$. We use the term relative interior, since the dimension of $S$ might be smaller than $m$. Let us denote the convex hull for $\left\{x_{i}\right\}_{i=1}^{n}$ by $\operatorname{conv}\left(\left\{x_{i}\right\}_{i=1}^{n}\right)$. Similarly, we can denote the convex hull for the positions of the neighbors of agent $i$ as $\operatorname{conv}\left(\left\{x_{j}\right\}_{j \in \mathcal{N}_{i}}\right)$.

Suppose Assumption 1 is fulfilled. We consider the case when

$$
V\left(x_{i}\right)=x_{i}^{T} x_{i} \quad \text { and } \quad W\left(x_{i}, x_{j}\right)=\left(x_{j}-x_{i}\right)^{T}\left(x_{j}-x_{i}\right) .
$$




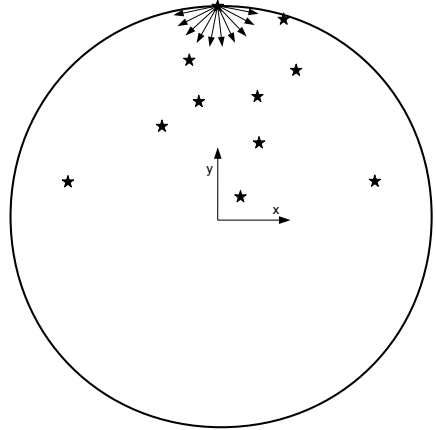

Fig. 1. $m=2$. The positions of the agents at a time $t$ are denoted by stars.

Suppose the functions in $\mathcal{F}$ are Lipschitz in $x$ on $\mathbb{R}^{m n}$, uniformly with respect to $t$, and continuous in $t$. Furthermore, suppose $V$ fulfills Assumption 2, then in Lemma 2 we can choose $\widehat{\beta}_{1}\left(\left\|x_{i}\right\|\right)=\widehat{\beta}_{2}\left(\left\|x_{i}\right\|\right)=\left\|x_{i}\right\|^{2}$, and obtain the result that any closed ball $\bar{B}_{r, m}$ in $\mathbb{R}^{m}$ is invariant and we can choose $\mathcal{D}=\mathcal{D}^{*}(\infty)=\left(\bar{B}_{r, m}\right)^{n}$. The point $x=0$ is uniformly stable. Thus, by Theorem 1 we obtain the result that if $\mathcal{G}_{\sigma(t)}$ is uniformly strongly connected, then $\mathcal{A}$ is globally quasiuniformly attractive relative to $\mathcal{D}^{*}(\infty)$. Unless $x_{i}=x_{j}$ for all $j \in \mathcal{N}_{i}$, for any agent $i$ that is furthest away from the origin, $f_{i}(t, x)$ as an element of the tangent space $T_{x_{i}} \mathbb{R}^{m}$ is inward-pointing on the boundary of the closed ball with radius equal to the norm of agent $i$. This is illustrated in Figure 1.

Suppose not only that $V$ fulfills Assumption 2, but also that $W$ fulfills Assumption 3. In this case, any closed ball in $\mathbb{R}^{m}$ is invariant and can be chosen as $\mathcal{D}^{*}(\infty)$, but also the largest Euclidean distance between any pair of agents is decreasing. This is illustrated in Figure 2. The solid circle denotes the boundary of the ball $\bar{B}_{r_{1}, 2}$ and the dashed circle denotes the boundary of the ball $\bar{B}_{r_{2}, 2}$, where

$$
r_{1}=\max _{k \in \mathcal{V}}\left\|x_{k}(t)\right\| \quad \text { and } \quad r_{2}=\max _{(k, l) \in \mathcal{V} \times \mathcal{V}}\left\|x_{k}(t)-x_{l}(t)\right\| .
$$

The dashed line denotes the distance between the two agents that are furthest away from each other.

Now Theorem 2 can be used to show that $\mathcal{A}$ is globally uniformly asymptomatically stable relative to $\mathcal{D}^{*}(\infty)$ if and only if $\mathcal{G}_{\sigma(t)}$ is uniformly quasi-strongly connected. For agent $i$, if $f_{i}(t, x)$ is inward-pointing relative to the convex hull of its neighbors [25], [26], then these conditions are fulfilled.

As a special case let

$$
f_{i}(t, x)=\sum_{j \in \mathcal{N}_{i}^{\sigma_{i}}(t)} a_{i j}\left(t-\gamma_{\sigma_{i}}(t)\right)\left(x_{j}-x_{i}\right),
$$

where $\alpha_{i j}(t)>0$ is continuous, positive and bounded for all $t$. Let us construct the set of functions $\mathcal{F}$ in the following way. There are $2^{n^{2}-n}$ graphs. For the $k$-th graph we define a corresponding function

$$
\begin{aligned}
& \tilde{f}_{k}(t, x) \\
= & {\left[\sum_{j \in \mathcal{N}_{1}} \alpha_{i j}(t)\left(x_{j}-x_{1}\right)^{T}, \ldots, \sum_{j \in \mathcal{N}_{n}} \alpha_{i j}(t)\left(x_{j}-x_{n}\right)^{T}\right]^{T}, }
\end{aligned}
$$

where (by a slight abuse of notation) $\mathcal{N}_{i}$ in this context is the

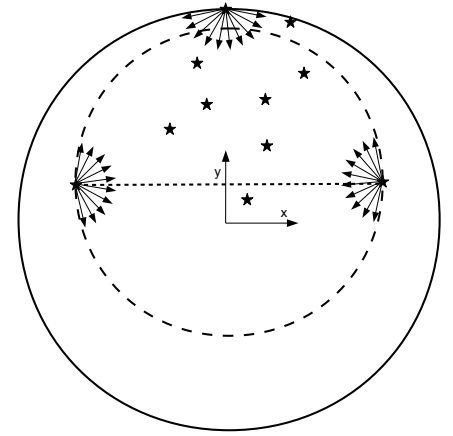

Fig. 2. $m=2$. The positions of the agents at time $t$ are denoted by stars.

neighborhood of agent $i$ in the graph $k$-th graph. Now we let

$$
\mathcal{F}=\left\{\tilde{f}_{k}\right\}_{k=1}^{2^{n^{2}-n}} .
$$

Now, using the functions

$$
V\left(x_{i}\right)=x_{i}^{T} x_{i} \quad \text { and } \quad W\left(x_{i}, x_{j}\right)=\left(x_{j}-x_{i}\right)^{T}\left(x_{j}-x_{i}\right),
$$

one can show global uniform asymptotic consensus relative to $\mathcal{D}^{*}(\infty)$.

\section{B. Consensus on $S O(3)$ using the Axis-Angle Representation}

Consider a system of $n$ rotation matrices in $S O(3)$ (controlled on a kinematic level) that asymptotically will reach consensus in the rotation matrices. For a rotation matrix $R_{i}$ there is a corresponding vector $x_{i}$, referred to as the Axis-Angle Representation of $R_{i}$. Locally around the identity matrix, in terms of kinematics we have that

$$
\dot{R}_{i}=R_{i} \widehat{\omega}_{i} \quad \text { or } \quad \dot{x}_{i}=L_{x_{i}} \omega_{i}
$$

where

$$
L_{x_{i}}=I_{3}+\frac{\widehat{x}_{i}}{2}+\frac{1}{\left\|x_{i}\right\|^{2}}\left(1-\frac{\operatorname{sinc}\left(\left\|x_{i}\right\|\right)}{\operatorname{sinc}^{2}\left(\frac{\left\|x_{i}\right\|}{2}\right)}\right) \widehat{x}_{i}^{2},
$$

and $\widehat{\omega}_{i}, \widehat{x}_{i}$ are the skew-symmetric matrices generated by $\omega_{i}, x_{i} \in \mathbb{R}^{3}$, respectively. We require that $x_{i}\left(t_{0}\right) \in B_{\pi, 3}$ for all $i$. Now we consider the case when

$$
\omega_{i}=\sum_{j \in \mathcal{N}_{i}(t)} \alpha_{i j}\left(t-\gamma_{\sigma_{i}}(t)\right)\left(x_{j}-x_{i}\right),
$$

where the function $\alpha_{i j}(t)$ is continuous, positive and bounded, and $\sigma \in \mathcal{S}_{\mathcal{F}}$. The set $\mathcal{F}$ is constructed in the same manner as outlined in the example in the previous section. The symmetric part of the matrix $L_{x_{i}}$ is positive definite on $B_{\pi, 3}$, and the system is at an equilibrium if and only if $x=\left(x_{1}, x_{2}, \ldots, x_{n}\right)^{T} \in \mathcal{A}$.

Let $V\left(x_{i}\right)=x_{i}^{T} x_{i}$. By observing that $x_{i}^{T} L_{x_{i}}=x_{i}^{T}$, it is easy to show that Assumption 2 holds for $V$. We can apply Lemma 2 with $\widehat{\beta}_{1}\left(\left\|x_{i}\right\|\right)=\widehat{\beta}_{2}\left(\left\|x_{i}\right\|\right)=\left\|x_{i}\right\|^{2}$, and show that any ball $\bar{B}_{r, 3}$ is invariant for $r<\pi$ and can be chosen to $\mathcal{D}=\mathcal{D}^{*}(\infty)$. Now, by using Theorem 1 , we can conclude that if the graph $\mathcal{G}_{\sigma(t)}$ is uniformly strongly connected, then $\mathcal{A}$ is globally quasi-uniformly attractive. 
In Figure 3 we illustrate the convergence of the $x_{i}$-states to consensus when controller (4) is used. In this simulation the $a_{i j}$-weights were constant, i.e., they are not time-varying. The dwell-time between consecutive switches was bounded from below by 0.25 . The left sub figure shows the time evolution of $\left\|x_{j}(t)-x_{1}(t)\right\|$ for all $i$. The right sub figure shows the time evolution of $[1,0,0]\left(x_{j}(t)-x_{1}(t)\right)$, i.e., the first element of $\left(x_{j}(t)-x_{1}(t)\right)$ for all $i$.
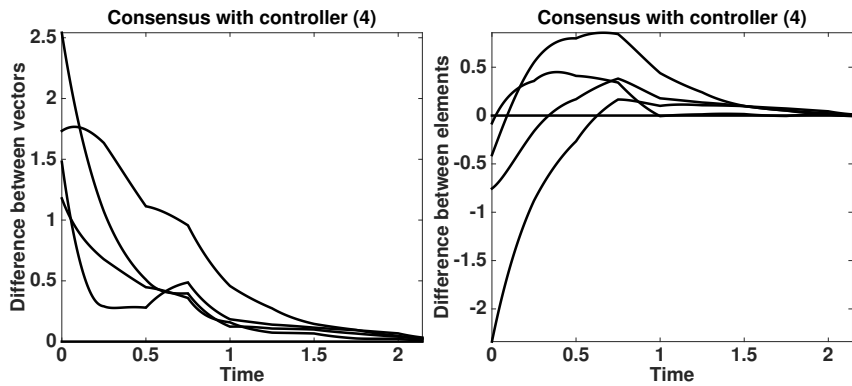

Fig. 3. Convergence to consensus for controller (4).

\section{PROOFS AND OUTLINE OF PROOFS}

In this section, first the explicit proofs for Lemma 1 and Lemma 2 are provided. Then outlines for the proofs of Theorem 1 and Theorem 1 are provided.

\section{A. Proofs for the lemmas}

Proof of Lemma 1: Since $\mathcal{D}$ is compact, we only need to verify that $\mathcal{D}^{*}(\tilde{t})$ is closed in order to show that $\mathcal{D}^{*}(\tilde{t})$ is compact. Suppose there is $x_{0} \notin \mathcal{D}^{*}(\tilde{t})$, such that there is a sequence $\left\{x_{0}^{i}\right\}_{i=1}^{\infty}$ that converges to $x_{0}$, where each element in the sequence is in $\mathcal{D}^{*}(\tilde{t})$. We would like to obtain a contradiction by showing that the solution $x^{\sigma}\left(t, t_{0}, x_{0}\right)$ does exist in $\mathcal{D}$ on the interval $\left[t_{0}, t_{0}+\tilde{t}\right)$ for any $t_{0}$, and $\sigma \in \mathcal{S}_{\mathcal{F}}$.

By using the fact that $\mathcal{D}$ is compact and that the righthand side of (1) is uniformly Lipschitz in $x$ on $\mathcal{D}$ and piecewise continuous in $t$, we can use the Continuous Dependency Theorem of initial conditions in order to guarantee that $\left\{x^{\sigma}\left(t, t_{0}, x_{0}^{i}\right)\right\}_{i=1}^{\infty}$ is a Cauchy sequence for arbitrary $t \in\left[t_{0}, t_{0}+\tilde{t}\right)$. Now we know, since $\mathcal{D}$ is compact, that $x^{*}(t)=\lim _{i \rightarrow \infty} x^{\sigma}\left(t, t_{0}, x_{0}^{i}\right)$ exists and $x^{*}(t) \in \mathcal{D}$. We want to prove that $x^{*}(t)$ is the solution for (1) on $\left[t_{0}, t_{0}+\tilde{t}\right)$ for the given $\sigma, t_{0}$ and $x_{0}$.

$$
\begin{aligned}
x^{*}(t) & =\lim _{i \rightarrow \infty} x^{\sigma}\left(t, t_{0}, x_{0}^{i}\right) \\
& =\lim _{i \rightarrow \infty} \int_{t_{0}}^{t} f\left(s, x^{\sigma}\left(s, t_{0}, x_{0}^{i}\right)\right) d s \\
& =\int_{t_{0}}^{t} \lim _{i \rightarrow \infty} f\left(s, x^{\sigma}\left(s, t_{0}, x_{0}^{i}\right)\right) d s \\
& =\int_{t_{0}}^{t} f\left(s, x^{*}(s)\right) .
\end{aligned}
$$

Hence, $x^{*}(t)$ is contained in $\mathcal{D}$ for all $t$ and $x^{*}(t)$ is a solution for (1). But since $\sigma$ and $t_{0}$ were arbitrary, it follows that $x_{0} \in$ $\mathcal{D}^{*}(\tilde{t})$, which is a contradiction.
Now we prove the statement that $\mathcal{D}^{*}(\infty)$ is invariant. Suppose $x_{0} \in \mathcal{D}^{*}(\infty)$ is arbitrary and let

$$
y=x^{\sigma^{\prime}}\left(t_{1}, t_{0}, x_{0}\right)
$$

for $\sigma^{\prime} \in \mathcal{S}_{\mathcal{F}}$ and $t_{1} \geq t_{0}$. Consider $x^{\sigma^{\prime \prime}}\left(t, t_{1}^{\prime}, y\right)$ for some arbitrary $\sigma^{\prime \prime} \in \mathcal{S}_{\mathcal{F}}$ and $t_{1}^{\prime}$. We need to show that $x^{\sigma^{\prime \prime}}\left(t, t_{1}^{\prime}, y\right)$ is contained in $\mathcal{D}$ for all $t \geq t_{1}^{\prime}$.

We define

$$
\sigma(t)= \begin{cases}\sigma^{\prime}\left(t-\left(t_{1}^{\prime}-t_{1}\right)\right) & \text { if } t<t_{1}^{\prime} \\ \sigma^{\prime \prime}(t) & \text { if } t \geq t_{1}^{\prime}\end{cases}
$$

which is contained in $\mathcal{S}_{\mathcal{F}}$. Thus

$$
x^{\sigma^{\prime \prime}}\left(t, t_{1}^{\prime}, y\right)=x^{\sigma}\left(t, t_{0}+\left(t_{1}^{\prime}-t_{1}\right), x_{0}\right),
$$

which is contained in $\mathcal{D}$ for all $t \geq t_{0}$ since $x_{0} \in \mathcal{D}^{*}(\infty)$. Thus, $y \in \mathcal{D}^{*}(\infty)$.

Proof of Lemma 2: In this proof, for ease of notation, we omit to denote the explicit dependence on $\sigma$ for $x$.

Since the origin is an interior point of $\mathcal{D}$, there is a ball $B_{\epsilon, m}$ such that $\left(B_{\epsilon, m}\right)^{n} \subset \mathcal{D}$ and $\epsilon>0$. Let $x_{0}=$ $\left[x_{10}^{T}, x_{20}^{T}, \ldots, x_{n 0}^{T}\right]^{T}$. Suppose $x_{0} \in\left(B_{\epsilon, m}\right)^{n}$, then there is a closed ball

$$
\bar{B}_{\epsilon^{\prime}, m n}\left(x_{0}\right) \subset\left(B_{\epsilon, m}\right)^{n}
$$

with $\epsilon^{\prime}>0$. Now according to Theorem 3.1. in [28], there is a $\delta^{\prime}>0$ such that the system has a unique solution $x\left(t, t_{0}, x_{0}\right)$ on $\left[t_{0}, t_{0}+\delta^{\prime}\right]$ in $\mathcal{D}$. We choose $\left[t_{0}, t_{0}+T^{\prime}\right)$ as the maximal half-open interval of existence of the unique solution. We know there are class $\mathcal{K}$ functions $\beta_{1}$ and $\beta_{2}$ such that

$$
\beta_{1}(\|y\|) \leq V(y) \leq \beta_{2}(\|y\|)
$$

for $y \in \mathbb{R}^{m}$.

Now, by using property (2) of Assumption 2 we get from the Comparison Lemma (Lemma 3.4 in [28]), that

$$
\max _{i \in \mathcal{V}}\left\{\left\|x_{i}(t)\right\|\right\} \leq \max _{i \in \mathcal{V}}\left\{\left\|x_{0 i}\right\|\right\}
$$

for $t \in\left[t_{0}, t_{0}+T^{\prime}\right)$. Now let $\delta=\beta_{2}^{-1}\left(\beta_{1}(\epsilon)\right)$. We suppose that $x_{0}$ was chosen such that

$$
x_{i}\left(t_{0}\right) \in \bar{B}_{\delta, m} \subset \bar{B}_{\epsilon, m} \text { for all } i \text {. }
$$

It follows that for $t \in\left[t_{0}, t_{0}+T^{\prime}\right)$,

$$
\begin{aligned}
& \max _{i \in \mathcal{V}}\left\{\left\|x_{i}(t)\right\|\right\}=\beta_{1}^{-1}\left(\beta_{1}\left(\max _{i \in \mathcal{V}}\left\{\left\|x_{i}(t)\right\|\right\}\right)\right) \\
& =\beta_{1}^{-1}\left(\max _{i \in \mathcal{V}}\left\{\beta_{1}\left(\left\|x_{i}(t)\right\|\right)\right\}\right) \leq \beta_{1}^{-1}\left(\max _{i \in \mathcal{V}}\left\{\left\|x_{i}(t)\right\|\right\}\right) \\
& \leq \beta_{1}^{-1}\left(\max _{i \in \mathcal{V}}\left\{\left\|x_{i}\left(t_{0}\right)\right\|\right\}\right) \leq \beta_{1}^{-1}\left(\max _{i \in \mathcal{V}}\left\{\beta_{2}\left(\left\|x_{i}\left(t_{0}\right)\right\|\right)\right\}\right) \\
& \leq \beta_{1}^{-1}\left(\beta_{2}\left(\max _{i \in \mathcal{V}}\left\{\left(\left\|x_{i}\left(t_{0}\right)\right\|\right)\right\}\right)\right) \leq \beta_{1}^{-1}\left(\beta_{2}(\delta)\right)=\epsilon .
\end{aligned}
$$

Now it follows by using Theorem 3.3 in [28], that the solution will stay in $\left(\bar{B}_{\epsilon, m}\right)^{n}$ for arbitrary times larger than $t_{0}$, i.e., $T^{\prime}=\infty$. 


\section{$B$. outline of the proofs of the theorems}

Outlines of the proofs of Theorem 1 and Theorem 2 are described here as a series of consecutive steps. We begin with Theorem 1.

\section{Outline of the proof of Theorem 1}

Step 1. The first step is to prove Lemma 3 below. In the lemma it is assumed that $\sigma \in \mathcal{S}_{\mathcal{F}}$ is fixed. Hence, for ease of notation, we omit to denote the explicit dependence on $\sigma$ for $x$.

Lemma 3. Suppose $x_{0}=\left[x_{10}^{T}, x_{20}^{T}, \ldots, x_{n 0}^{T}\right]^{T} \in \mathcal{A}^{c} \cap \mathcal{D}^{*}(\infty)$ and Assumption 2 2) holds. Suppose there is a non-negative function $\beta(y, \tilde{t})$, which is increasing in $\tilde{t}$ for $y \in \mathcal{A}^{c} \cap \mathcal{D}^{*}(\infty)$. Furthermore, suppose that for $y \in \mathcal{A}^{c} \cap \mathcal{D}^{*}(\infty)$ there is $\tilde{t}^{\prime}(y)>0$ such that for $\tilde{t} \geq \tilde{t}^{\prime}(y)$ it holds that $\beta(y, \tilde{t})>0$.

$$
\text { If }
$$

$$
\max _{i \in \mathcal{V}}\left\{V\left(x_{i}\left(t_{0}+\tilde{t}, t_{0}, x_{0}\right)\right)\right\}-\max _{i \in \mathcal{V}}\left\{V\left(x_{i 0}\right)\right\} \leq-\beta\left(x_{0}, \tilde{t}\right),
$$

then $x(t) \rightarrow \mathcal{A}$ as $t \rightarrow \infty$ for all $t_{0}$.

Furthermore, if $\beta$ is lower semi-continuous in $y$, and $\tilde{t}^{\prime}$ is independent of $y$, then $\mathcal{A}$ is globally quasi-uniformly attractive relative to $\mathcal{D}^{*}(\infty)$.

To prove the statement in the lemma we use the positive limit set $L^{+}\left(x_{0}, t_{0}\right)$ of the solution $x\left(t, t_{0}, x_{0}\right)$ when $x_{0} \in \mathcal{D}^{*}(\infty)$. This limit set exists and is compact, and $x(t)$ approaches it as the time goes to infinity, however we do not guarantee that it is invariant, which is the case for an autonomous system. The set $L^{+}\left(x_{0}, t_{0}\right)$ is contained in $\mathcal{D}^{*}(\infty)$, so any alternative solution of (1) that starts in $L^{+}\left(x_{0}, t_{0}\right)$ will remain in $\mathcal{D}^{*}(\infty)$.

Step 2. In this step Lemma 4 below is proven.

Lemma 4. Suppose that Assumption 1 and Assumption 2 2), 3) hold, $\sigma \in \mathcal{S}_{\mathcal{F}}, x^{\sigma}\left(t_{0}\right) \in \mathcal{D}^{*}(\infty) \cap \mathcal{A}^{c}$ and $\mathcal{G}_{\sigma(t)}$ is uniformly strongly connected. If $t_{0}$ is a switching time of $\sigma$, it follows that

$$
\max _{i \in \mathcal{V}}\left\{V\left(x_{i}^{\sigma_{i}}(t)\right)\right\}-\max _{i \in \mathcal{V}}\left\{V\left(x_{i}^{\sigma_{i}}\left(t_{0}\right)\right)\right\}<0
$$

for any $t \geq n\left(T^{\sigma}+2 \tau_{D}\right)+t_{0}$, where $T^{\sigma}$ is given in Definition 8 and $\tau_{D}$ is the lower bound on the dwell-time between two consecutive switches of the switching signals in $\sigma$.

Step 3. In this step we prove the theorem by showing that there is a function $\beta$ with the properties given in Lemma 3. For each $\sigma \in \mathcal{S}_{\mathcal{F}}$, there is a corresponding $\beta$. The key idea is to use Lemma 4 on the one hand, and the fact that the dwell-time between consecutive switches is bounded on the other. The solution depends continuously on the switching times, which, due to the bounded dwell-time assumption, are contained in a compact set. Thus, the supremum of the expression in the left-hand side of (5) is strictly negative, which in turn can be used to show the existence of a $\beta$-function that we seek.

\section{Outline of the proof of Theorem 2}

The procedure to prove the theorem is in the spirit of the proof of Theorem 1, with some key differences.
Step 1. We prove Lemma 5 below. Similar to Lemma 3, it is assumed that $\sigma \in \mathcal{S}_{\mathcal{F}}$ is fixed. Hence, for ease of notation, we omit to denote the explicit dependence on $\sigma$ for $x$.

Lemma 5. Suppose that $x_{0}=\left[x_{10}^{T}, x_{20}^{T}, \ldots, x_{n 0}^{T}\right]^{T} \in \mathcal{A}^{c} \cap$ $\mathcal{D}^{*}(\infty)$ and Assumption 3 1), 2) hold. Let

$$
\beta(y, \tilde{t}): \mathbb{R}^{+} \times \mathbb{R}^{+} \rightarrow \mathbb{R}^{+}
$$

be increasing in $\tilde{t}$ and lower semi-continuous in $y$. Furthermore, suppose that there is $\tilde{t}^{\prime}>0$, such that for $\tilde{t} \geq \tilde{t}^{\prime}$, it holds that $\beta(y, \tilde{t})>0$ for all positive $y$.

If

$$
\begin{aligned}
& \max _{i, j \in \mathcal{V}}\left\{W\left(x_{i}\left(t, t_{0}, x_{0}\right), x_{j}\left(t, t_{0}, x_{0}\right)\right)\right\} \\
- & \max _{i, j \in \mathcal{V}}\left\{W\left(x_{i}\left(x_{0}\right), x_{j}\left(x_{0}\right)\right)\right\} \leq-\beta\left(\operatorname{dist}\left(x_{0}, \mathcal{A}\right), t-t_{0}\right),
\end{aligned}
$$

then $\mathcal{A}$ is globally uniformly asymptotically stable relative to $\mathcal{D}^{*}(\infty)$.

Step 2. In this step Lemma 6 below is proven.

Lemma 6. Suppose Assumption 1 and Assumption 3 1), 2), and 3) hold, $x_{0} \in \mathcal{D}^{*}(\infty) \cap \mathcal{A}^{c}$ and $\sigma \in \mathcal{S}_{\mathcal{F}}$. Furthermore, suppose $\mathcal{G}_{\sigma(t)}$ is uniformly quasi-strongly connected, then

$$
\max _{i, j \in \mathcal{V}}\left\{W\left(x_{i}^{\sigma_{i}}(t), x_{j}^{\sigma_{i}}(t)\right)\right\}-\max _{i, j \in \mathcal{V}}\left\{W\left(x_{i 0}, x_{j 0}\right)\right\}<0
$$

if $t_{0}$ is a switching time and $t \geq n\left(T^{\sigma}+2 \tau_{D}\right)+t_{0}$, where $T^{\sigma}$ is given in Definition 8 and $\tau_{D}$ is the lower bound on the dwell-time between two consecutive switches of the switching signals in $\sigma$.

Step 3. Now the "if-part" of the theorem is proved. The approach resembles that of step 3 in the proof of Theorem 1 . We use Lemma 6 together with the bounded dwell-time assumption to show that there is a $\beta$-function that satisfies the assumptions in Lemma 5.

Step 4. Now the "only if-part" of the theorem is proved by a contradiction argument. If the graph is not quasi-strongly connected we show that we get a contradiction to the assumption that the convergence to $\mathcal{A}$ is uniform. We do not actually disprove the statement that the convergence to $\mathcal{A}$ occurs. But if such convergence occurs, it cannot be uniform.

\section{CONCLUSIONS AND OUTLOOK}

This note presented two theorems for consensus in nonlinear continuous-time multi-agent systems with switching timevarying interconnection topologies. The two theorems are along the lines of Lypunov's second method for consensus. A key point is that the Lyapunov-like functions are local. They are either functions of the individual states of the agents, Theorem 1, or functions of pairs of states, Theorem 2. This local structure can simplify the convergence analysis, as the state of the whole system does not need to be considered only individual states, pairs of states, and neighboring states.

In industrial applications there are other issues than switching topologies. Those include time-delays, packet dropouts or quantization [29], [30]. It would be interesting to 
explore the possibilities of augmenting the presented theory in such directions.

\section{REFERENCES}

[1] C. Bergenhem, Q. Huang, A. Benmimoun, and T. Robinson. Challenges of platooning on public motorways. In 17th World Congress on Intelligent Transport Systems, pages 1-12, 2010.

[2] A. Möller, U.T. Jönsson, A. Blomgren, and F. Gunnarsson. Stability of rate and power control algorithms in wireless cellular networks. In $C D C$-ECE, pages 4535-4541, 2011.

[3] B.Ø. Palsson. Systems biology: simulation of dynamic network states. Cambridge University Press, 2011.

[4] R. Olfati-Saber and R. M. Murray. Consensus problems in networks of agents with switching topology and time-delays. Automatic Control, IEEE Transactions on, 49(9):1520-1533, 2004.

[5] M. Mesbahi and M. Egerstedt. Graph theoretic methods in multiagent networks. Princeton University Press, 2010.

[6] L. Schenato and F. Fiorentin. Average timesynch: A consensusbased protocol for clock synchronization in wireless sensor networks. Automatica, 47(9):1878-1886, 2011.

[7] C. W. Reynolds. Flocks, herds and schools: A distributed behavioral model. In ACM SIGGRAPH Computer Graphics, volume 21, pages 25-34. ACM, 1987.

[8] T. Vicsek, A. Czirok, B-J. Eshel, I. Cohen, and O. Shochet. Novel type of phase transition in a system of self-driven particles. Physical Review Letters, 75(6):1226, 1995.

[9] L. Moreau. Stability of multiagent systems with time-dependent communication links. IEEE Transactions on Automatic Control, 50(2):169-182, 2005.

[10] R. Olfati-Saber, J.A. Fax, and R.M. Murray. Consensus and cooperation in networked multi-agent systems. Proceedings of the IEEE, 95(1):215233, 2007.

[11] W. Ren and R. W. Beard. Consensus seeking in multiagent systems under dynamically changing interaction topologies. IEEE Transactions on Automatic Control, 50(5):655-661, 2005.

[12] W. Ren and R.W. Beard. Distributed consensus in multi-vehicle cooperative control: theory and applications. Springer, 2008.

[13] F. Garin and L. Schenato. A survey on distributed estimation and control applications using linear consensus algorithms. In Networked Control Systems, pages 75-107. Springer, 2010.

[14] W. Ren, R. W. Beard, and E.M. Atkins. A survey of consensus problems in multi-agent coordination. In American Control Conference, 2005. Proceedings of the 2005, pages 1859-1864. IEEE, 2005.

[15] Y. Cao, W. Yu, W. Ren, and G. Chen. An overview of recent progress in the study of distributed multi-agent coordination. Industrial Informatics, IEEE Transactions on, 9(1):427-438, 2013.

[16] J. Thunberg, J. Goncalves, and X. Hu. Consensus and formation control on se (3) for switching topologies. Automatica, 66:109-121, 2016.

[17] E. Montijano, J. I. Montijano, and C. Sagues. Fast distributed consensus with chebyshev polynomials. In American Control Conference (ACC), 2011, pages 5450-5455. IEEE, 2011.

[18] L. Xiao, S. Boyd, and S-J. Kim. Distributed average consensus with least-mean-square deviation. Journal of Parallel and Distributed Computing, 67(1):33-46, 2007.

[19] L. Moreau. Stability of continuous-time distributed consensus algorithms. In IEEE Conference on Decision and Control, volume 4, pages 3998-4003. IEEE, 2004.

[20] R. Olfati Saber and R.M. Murray. Consensus protocols for networks of dynamic agents. In Proceedings of the American Control Conference. 2003.

[21] C-Q. Ma and J-F. Zhang. Necessary and sufficient conditions for consensusability of linear multi-agent systems. IEEE Transactions on Automatic Control, 55(5):1263-1268, 2010.

[22] Y. Yuan, G-B. Stan, L. Shi, M. Barahona, and J. Gonçalves. Decentralised minimum-time consensus. Automatica, 49(5):1227-1235, 2013.

[23] E. Montijano, J. Thunberg, X. Hu, and C. Sagues. Epipolar visual servoing for multirobot distributed consensus. IEEE Transactions on Robotics, 29(5):1212-1225, 2013.

[24] J. Thunberg, W. Song, E. Montijano, Y. Hong, and X. Hu. Distributed attitude synchronization control of multi-agent systems with switching topologies. Automatica, 50(3):832-840, 2014.

[25] B. Francis Z. Lin and M. Maggiore. State agreement for continuous-time coupled nonlinear systems. SIAM Journal on Control and Optimization, 46(1):288-307, 2007
[26] G. Shi and Y. Hong. Global target aggregation and state agreement of nonlinear multi-agent systems with switching topologies. Automatica, 45(5):1165-1175, 2009.

[27] B. Afsari. Riemannian Lp center of mass: Existence, uniqueness and convexity. In Proc. Amer. Math. Soc, volume 139, pages 655-673, 2011.

[28] H. K. Khalil. Nonlinear systems, volume Third Edition. Prentice hall, 2002.

[29] H. Li, C. Wu, P. Shi, and Y. Gao. Control of nonlinear networked systems with packet dropouts: interval type-2 fuzzy model-based approach. IEEE Transactions on Cybernetics, 45(11):2378-2389, 2015.

[30] T. Wang, H. Gao, and J. Qiu. A combined adaptive neural network and nonlinear model predictive control for multirate networked industrial process control. IEEE Transactions on Neural Networks and Learning Systems, 27(2):416-425, 2016.

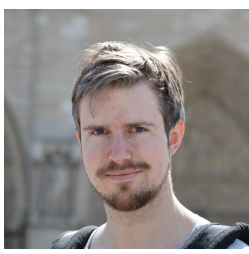

Johan Thunberg Johan Thunberg received the M.Sc. and Ph.D. degrees from KTH Royal Institute of Technology, Sweden, in 2008 and 2014, respectively. Between 2007 and 2008 he worked as a research assistant at the Swedish Defence Research agency (FOI) and between 2008 and 2009 he worked as a programmer at ENEA AB. Currently he is an AFR/FNR postdoctoral research fellow at the Luxembourg Centre for Systems Biomedicine, University of Luxembourg.

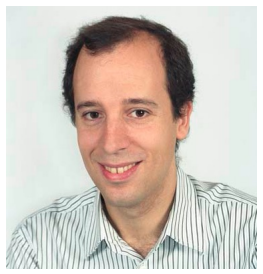

Jorge Goncalves Jorge Goncalves is currently a Professor at the Luxembourg Centre for Systems Biomedicine, University of Luxembourg and a Principal Research Associate at the Department of Engineering, University of Cambridge. He received his Licenciatura (5-year S.B.) degree from the University of Porto, Portugal, and the M.S. and Ph.D. degrees from the Massachusetts Institute of Technology, Cambridge, MA, all in Electrical Engineering and Computer Science, in 1993, 1995, and 2000, respectively. He then held two postdoctoral positions, first at the Massachusetts Institute of Technology for seven months, and from 2001 to 2004 at the California Institute of Technology with the Control and Dynamical Systems Division. At the Information Engineering Division of the Department of Engineering, University of Cambridge he was a Lecturer from 2004 until 2012, a Reader from 2012 until 2014, and since 2014 he is a Principal Research Associate. From 2005 until 2014 he was a Fellow of Pembroke College, University of Cambridge. From June to December 2010 and January to September 2011 he was a visiting Professor at the University of Luxembourg and California Institute of Technology, respectively. Since 2013 he is a Professor at the Luxembourg Centre for Systems Biomedicine, University of Luxembourg.

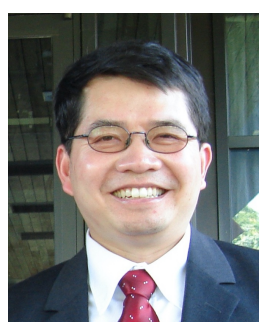

Xiaoming Hu Xiaoming Hu received the B.S. degree from the University of Science and Technology of China in 1983, and the M.S. and Ph.D. degrees from the Arizona State University in 1986 and 1989 respectively. He served as a research assistant at the Institute of Automation, the Chinese Academy of Sciences, from 1983 to 1984 . From 1989 to 1990 he was a Gustafsson Postdoctoral Fellow at the Royal Institute of Technology, Stockholm, where he is currently a professor of Optimization and Systems Theory. His main research interests are in nonlinear control systems, nonlinear observer design, sensing and active perception, motion planning, control of multi-agent systems, and mobile manipulation. 